\title{
Integrated and Networked Systems and Processes-A Perspective for Digital Transformation in Thermal Process Engineering
}

\author{
Michael Maiwald (1) \\ Bundesanstalt für Materialforschung und -prüfung (BAM), 12489 Berlin, Germany; michael.maiwald@bam.de; \\ Tel.: +49-30-8104-1140
}

Received: 24 October 2019; Accepted: 20 February 2020; Published: 4 March 2020

\begin{abstract}
Separation technology as a sub-discipline of thermal process engineering is one of the most critical steps in the production of chemicals, essential for the quality of intermediate and end products. The discipline comprises the construction of facilities that convert raw materials into value-added products along the value chain. Conversions typically take place in repeated reaction and separation steps-either in batch or continuous processes. The end products are the result of several production and separation steps that are not only sequentially linked, but also include the treatment of unused raw materials, by-products and wastes. Production processes in the process industry are particularly susceptible to fluctuations in raw materials and other influences affecting product quality. This is a challenge, despite increasing fluctuations, to deliver targeted quality and simultaneously meet the increasing dynamics of the market, at least for high value fine chemicals. In order to survive successfully in a changed environment, chemical companies must tread new paths. This includes the potential of digital technologies. The full integration and intelligent networking of systems and processes is progressing hesitantly. This contribution aims to encourage a more holistic approach to the digitalization in thermal process engineering by introduction of integrated and networked systems and processes.
\end{abstract}

Keywords: smart sensors; digitalization; digital transformation; process industry; thermal process engineering; digital twins

\section{Introduction}

Autonomous driving means the independent, targeted driving of a vehicle in real traffic, without the intervention of the driver. Although it represents a paradigm shift, there is technically an evolutionary process behind it: The successive introduction of interacting smart sensors, actuators and communication and their integration into machine-supported decision-making processes.

Sensors use cameras, radar or lasers to constantly record the surroundings and other information such as the speed, acceleration or location coordinates of the vehicle. An autonomous computer in the car fuses the sensor data. It is made up of the environmental situation of the vehicle. Automatic driving decisions are derived and passed on to the vehicle's actuators such as steering, engine control or brakes. Very large amounts of data must be handled here with short response times. The control algorithms are based on machine learning. Recurring complex tasks are broken down into subtasks, such as the recognition of traffic signs [1]. In preliminary stages of autonomous driving, the technology supports the human reaction of the driver.

The degree of maturity in an autonomous vehicle is therefore constantly increasing and is developing conventional driving to a semi-automated driving mode using driver assistance systems (cruise control, distance control, tracking systems), thereby increasing the added value. However, the 
driver must continue to drive with full attention. If the driver assistance systems are networked with each other, it is already possible to speak of highly automated systems. Although extreme situations are indicated and dangers are reduced, driver intervention is necessary. There is still a long way to reach full automation and fully autonomous driving, as described above.

Transposed to the autonomous chemical plant (see Figure 1), the current situation would be equivalent to classical PCS operation. Assisted operation (i.e., partial automation) is already made possible by Advanced Process Control (APC) in many cases. APC could be described as a suggestion scheme for process changes and is often used in exactly this way (traffic light function) and unfortunately not in direct loops. Semi-autonomous processes are states of maturity in which process changes in the design space already run fully automatically. This can be supported by soft sensing, i.e. virtual sensors that use dynamic process models in combination with ordinary plant data to provide information without the need for physical measurement. The encapsulation of sub-processes will also be of great benefit, for example for remote control of plants. Inside, it will become more complex, but easier for the user.

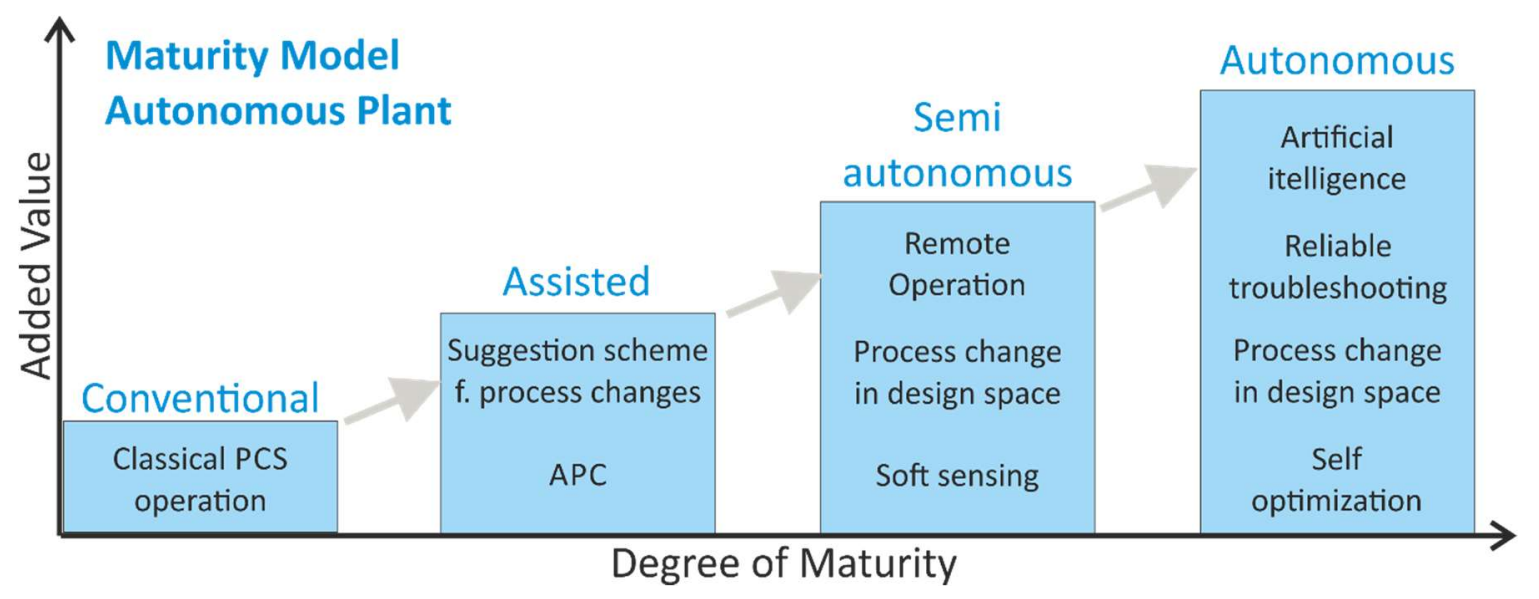

Figure 1. Increasing maturity of an autonomous plant (according to [2]).

This is why autonomous driving of process plants is also desired-naturally on the basis of networked smart sensors and actuators that continuously provide information about the processes and also feed big or smart data approaches. Field devices within smart modular production units are no longer single measuring points but display process states or generate new information by combining all available information.

As mentioned at the very beginning, the reliance on raw materials necessitates flexibility and optimum control of (thermal) processes, which typically do not yet exist today. The models, which are currently used for the plant design only, can be exploited in a first step in conjunction with sensors for closed loop control. The interaction of modular production systems, digital twin, smart field devices, and advanced process control/model-based control is explained in more detail below.

\section{Current Situation and Perspectives}

\subsection{Process Control}

Digitalization is also affecting separation technology. In the near future, reaction monitoring and process control will look different than at present. This section provides a brief overview of current developments in the field of process engineering, such as modern process design, integrated processes (e.g., reactive separation processes) and intensified processes as well as requirements for process control, model-based control and soft sensing. Finally, the current and future requirements for process automation will be briefly presented, as these are discussed for the future projects "Industry 4.0". The vision of a "Smart Factory" based on the Industrial Internet of Things (IIoT) enables a fourth 
industrial revolution also for the process industry, although there are differences to the manufacturing industry for which Industry 4.0 was developed. Future automation will be based on intelligent field devices, digital field networks, Internet Protocol (IP)-enabled connectivity and web services.

Today, optimized process design depends on computer-assisted tools that take into account mass transfer, thermodynamics, kinetics and other physical properties of the treated materials. Typically, a sufficient understanding of such properties exists and is implemented in dynamic numerical models. (However, many classical processes are not completely understood in this way.) Dynamic models are again the essential basis for optimized process and plant planning. Unfortunately, they are yet only sparsely used for process control—and actually even worse, that many important processing units do not have adequate dynamic models but only steady-state models.

An integrated process control approach enables constant product quality and prevents production failures by effectively compensating for process fluctuations. In a conventional approach, quality is determined by taking samples from material streams and performing offline analyses, called in-process control, at-line or off-line monitoring. By applying Quality by Design $(\mathrm{QbD})$ approaches, quality can be significantly improved to generate less waste, reduce the reprocessing of inferior material and produce products of the highest quality.

Digital transformation must now be designed in such a way that the dynamic models are not only used once for the design of a plant but are initially utilized continuously for operation in order to control and optimize in a model-based manner. Furthermore, the models are to be used as knowledge for further plants, although production plants are not at all similar for various reasons. Yet there is a chance to transfer principles and use them collaboratively. Finally, a way should be found to learn from the current production process and to constantly improve the models with the data obtained. Such a model container is called "Digital Twin". High quality data from proper sensors are most important to validate such "Digital Twins". Both lack of data and lack of high-quality data are currently the bottleneck for acceptable "Digital Twins" in process engineering.

A definition by Lee [3] brings this to the point: "A cyber-physical system (CPS) is an integration of computation with physical processes whose behavior is defined by both cyber and physical parts of the system. Embedded computers and networks monitor and control the physical processes, usually with feedback loops where physical processes affect computations and vice versa".

\subsection{Integrated Process Design and Intensified Processes}

Innovative explorations, developments and, concepts in the field of process engineering and in particular process intensification are currently promoted for the purpose of analyzing and designing innovative equipment and manufacturing concepts. This leads to a significant improvement in sustainability, efficiency, and environmental performance, alternative energy conversion, alternative transport mechanisms, intensified hydrodynamics, structured environments, multifunctionality, and increased plant operation.

\subsubsection{Intensified Continuous Processes}

Intensified continuous processes are in the spotlight of current research. Compared to conventional batch processes, intensified continuous production enables the introduction of new compounds that are difficult to produce, leads to better product uniformity and drastically reduces the amount of raw materials and energy required. With the same equipment, flexible (modular) chemical plants can produce different products with short downtimes between two campaigns and can quickly bring new products to market. These facilities are typically smaller in size than large basic chemical facilities, but are still able to produce kilograms to tons of specialty products daily. Such flexible (modular) plants can be provided in containers the size of freight containers.

Modular systems reduce complexity through encapsulation. As one of the first manifestations of industry 4.0 and cyber-physical systems, concepts for modular production are derived [4]. Modularization further increases the flexibility, availability and capacity utilization of plants, and 
different products can be brought to market more quickly. It is therefore also conceivable for module-based specialty chemicals to benefit from a dynamic model of the manufacturing industry and to realize shorter innovation and product cycles. The concept also allows higher quantities of more standardized field devices in modular systems.

However, full automation is a prerequisite for realizing such benefits from intensified continuous plants. In continuous flow processes, continuous automated measurements and strict closed-loop control of product quality are essential. If these are not available, there is a high risk that large quantities of products will be produced outside specification.

\subsubsection{Integrated Processes}

Another accomplishment of modern process engineering is the switch to integrated processes that unite reaction and separation in a single unit. This trend is simultaneously motivated by a number of advantages, such as the reduction of equipment and plant size as well as the improvement of process performance and thus a better process economy. Reactive distillation is an outstanding example of a reactive separation process. Especially for equilibrium reactions such as esterification, ester hydrolysis, or etherification, the combination of reaction and separation within a special zone of a reactive distillation column is a well-known alternative to the traditional processes with consecutive reaction and separation steps [5]. Typically, non-ideal aqueous-organic mixtures are formed which tend to form azeotropes which can be overcome by membrane separations (e.g., pervaporations or vapour permeations) [6]. On the other hand, undesired effects can also occur, such as increasing by-product formation. A hybrid process of membrane-supported reactive distillation thus contributes to a sustainable process improvement through the resulting synergy effects and enables the reduction of investment and operating costs. An overview of hybrid processes that combine pervaporation with one or more other separation technologies can be found in literature (see, e.g., $[7,8]$ ).

In the past few years, a significant number of papers have been issued containing well-documented information on laboratory-scale reactive distillation experiments, together with simulation results that are generally in accordance with the experimental data (see, e.g., [9-11]). The modelling and simulation of the reactive distillation process, carried out both with the equilibrium stage and the rate-based approach, is based on careful experimentation and modelling of the fundamental vapour-liquid and liquid-liquid equilibria, reaction equilibrium, and kinetics. It is shown that the separation side of the process can be well described with classical methods. In contrast, a correct description of the reaction kinetics under such conditions is complex because laboratory experiments show very different results than those from real process conditions. Understanding the reaction is the key to successful design and scale-up of heterogeneously catalyzed reactive distillation.

\section{Multi-Purpose Distillation Subsystems Lead to Increased Plant Flexibility-A Vision}

It has already become possible to operate and control some modules independently, such as refrigeration systems. Although complex control algorithms stand behind a refrigeration module and operation is critical for the entire process, modularity is no longer being questioned. The module only transmits status information, such as current cooling load, available cooling capacity, energy consumption, maintenance status, etc. to the process control system. In the future, it is obvious to break down many further unit operations, such as distillations, drying, etc., into separate units [8]. These Cyber-Physical Production Systems then control themselves independently via their smart sensors.

Distillation subsystems in process industries will be considered as separate unit operations in the future. The distillation parameters required for this flexibilization of the separation tasks to be performed are available in the network as parameter sets (material data and distillation models for a given material system).

If, for example, a distillation plant is not used to full capacity and there is a need for separation, the plant carries out the following steps autonomously: 
- The system procures the details of the separation process, such as reactant composition, product specification, required production quantities over time, etc. via the network.

- Based on its engineering specification and material compatibility information from the network, the plant checks whether the separation task can be performed in accordance with the specifications.

- The field devices and process sensors download the required application parameters from the network. In the case of a process spectrometer, for example, these are suitable chemometric models to determine the required compound concentrations. If the data sets are not available, a service provider is commissioned to create the required application under the given boundary conditions.

- The distillation plant obtains the necessary materials data (boiling curves, etc.) from the network which are required for parameterization of the plant. Models for initial simulations or feasibility tests do not have to be of high quality and can be obtained as apps. Models for superior production will presumably come from own resources and will probably already be available in the network today for standard separation tasks.

- The plant parameterizes itself independently and then optimizes itself during operation. In the case of large and sluggish plants, environmental and weather influences are also considered and weather forecasts are included.

\section{Requirements for Field Devices 4.0}

In the following, the requirements of process sensors for future automation concepts are described [8]. The most important advantages are

- Production modules, process sensors, and actuators provide additional information and services for defined addressees and generate new information from this in a network that goes beyond the sum of the information of the individual sensors.

- The smart functions of the automation components simplify their use and enable plug and play, even though they are becoming more complex. This is especially important for

- Self-diagnosis

- Self-calibration

- Self-configuration, c.f., self-parameterization

For process sensors and actuators to be an essential part of cyber-physical systems, they first need to fulfill the inherent task of measuring and presenting process information. The basic prerequisite for this is the performance of such automation components. This will constantly develop due to various technological constraints. The following essential statements can be made:

- The miniaturization progress continues (miniaturization allows the access of complex technology to processes).

- The spread of microelectromechanical systems, optical-electromechanical sensors is beyond special applications and can be used for smart sensor functions such as maintenance and operational functions.

- The computing power within the automation components increases. The price for this is decreasing (faster processors in the automation components, data evaluation, chemometrics on the automation components).

- Automation components become more autonomous (communication autonomy), energy autonomy through energy harvesting.

- Linking of automation components or their data leads to increased performance (informative value, reliability, measurement accuracy if necessary).

- A safe relapse strategy exists to avoid operational disturbances. 


\subsection{How Smart Are the Field Devices already Today?}

The benefits of smart field devices in the process industry and their communication options were described in more detail for the first time in 2015 in the technology roadmap "Process Sensors 4.0" [12] (see below), where it was predicted that complex measurement technology would already be introduced to processes today.

The roadmap explained typical features of smart field devices, which are explained in Figure 2 and below. Many of these requirements have now been successfully and reliably implemented and testify to the excellent cooperation between users in the process industry and their device and software manufacturers in committees and joint projects. Digital transformation is understood by all as a management task.

$\begin{array}{llll}\text { Connectivity and } & \text { Maintenance and } \\ \text { Communication Ability } & \text { Traceability and } \\ \text { Internal Functions } & \text { Compliance }\end{array}$

Figure 2. The most important smart functions of future process sensors according to [12].

\subsubsection{Connectivity and Communication Skills}

A uniform protocol and a uniform fieldbus are required for trouble-free communication between all automation components. Meanwhile the standard OPC Unified Architecture (OPC-UA, [13]) is considered to be set and can be regarded as a small triumph of industry 4.0. Non-Ethernet field buses are still dominant today against the background of a grown landscape in Brown Field applications and the often very special requirements for power supply and explosion protection.

OPC-UA is already used to connect automation components in the higher hierarchies, for example to ERP or production assistance systems. Increasingly, however, the standard will also be possible at device level (e.g., for smart laboratory devices [14]) and field level. While limited fieldbus network infrastructures still exist today, the process industry of the future will therefore be based on new network standards with the speed and flexibility of standard Ethernet and IP technologies, such as the standard Advanced Physical Layer (APL). At the same time, the computing power in the automation components at the field level is increasing in order to perform OPC UA communication and other computing operations in an energy-saving manner, such as encryption tasks or data analyses.

\subsubsection{Maintenance and Operational Functions}

The maintenance functions of the smart field devices now enable interactive assistance functions that greatly simplify implementation, calibration, troubleshooting or repair. These functions may or may not be included in the sensor itself. As a rule, the unambiguous assignment is made via an identifier on the field device (e.g., barcode, RFID, serial number) in order to retrieve data and service functions from the company's own asset database or from the manufacturer (industry 4.0 administration shell). Sensor access is granted via staggered access rights and user groups. Here it is an ongoing task for users and equipment manufacturers to create uniform standards for safety and functionality and to adapt them to current requirements. Among other things, ticket-based access rights instead of passwords are currently being discussed, as the latter are not manageable in an automation landscape of several thousand devices and against the background of changing personnel.

Because the maintenance and operational functions are of great benefit, some innovative companies in the process industry are currently covering their plants with additional network capabilities, mostly based on wireless technologies. Companies have also started to digitize their asset and asset plans completely. 
The data and service functions are operating instructions or videos for implementing, configuring, maintaining, calibrating, or repairing assets. Context information such as reference values, plant statuses or historical data can also be called up. If suitable, maintenance and operational functions can also be transferred to suitable third-party providers or the manufacturer, and invoices for field device costs or relevant services can be mapped in the ERP system.

\subsubsection{Traceability and Compliance}

The requirement for rights assignment and traceability of all changes to a field device is an important asset function and can be tracked via audit trails-ideally outside the sensor. Frequently, re-parameterizations or updates of the field device itself are still necessary if, for example, a product is changed or a current firmware is installed. This is difficult to map in a regulated environment (e.g., Good Manufacturing Practice, GMP). Very often security gaps exist via insufficiently protected maintenance channels.

Traceability and compliance are greatly simplified in the course of digitalization. In future, changes or updates to the field device can be tested and verified virtually before they are rolled out to the field device.

\subsubsection{Virtual Description}

Virtual writability supports the "end-to-end engineering" required by industry 4.0. The reference architecture model RAMI 4.0 [15] and the currently revised IEC 62890 (Life-Cycle Management) describe both this continuous engineering and further dimensions of information exchange very concretely. RAMI 4.0 is a key to understanding the different hierarchical levels in Industry 4.0.

In general, the use of digital plant planning tools makes digital, uniform descriptions of production and automation components increasingly necessary, and their technical data or functionalities must be available in the form of (dynamic) models. This information is in turn available to other hierarchical levels to simulate a production unit (and later even an entire factory), for example, before it is assembled and operated in its life cycle.

Consistent application of this concept is still outstanding today with regard to more complex production units, integrated processes or interrelated plants. The creation of a digital twin of an entire production unit or factory today still requires several years of development and validation time. For the controlled transfer of the information as a validated model to the operational instances, however, clearly defined functions and tiered access rights are required.

\subsubsection{Ability to Interact and Bidirectionality}

Inevitably the comparison with distributed networks, as derived in 1964 by engineer Paul Baran (1926-2011) for communication networks, becomes obvious [16]. Today, distributed networks are still the basis for the worldwide Internet and modern telecommunications. Instead of a fixed wire connection between the participants in centralized or decentralized networks, all participants log on to the system with their capabilities and are optimally utilized.

This image was the consistent translation of Industry 4.0 as flexible production for volatile markets with adaptive, self-configuring, self-organizing, flexible production systems with a high degree of networking and highly available information services-and the corresponding value-added contributions.

If we make these virtual connections thorough and secure, the advantages of digital transformation are mostly revealed. Distributed networks are more reliable, even if individual connections fail. Utilization is better distributed. Virtual operating systems and databases no longer fail-proportional to hardware investment based on a risk assessment. Migration of operating systems and compatibility of software and data did not need to scare us in this case in the future. 


\subsubsection{Logic Functions}

In a distributed network all participants login with their individual attributes. Particularly about their data storage and computing capacities in order to represent logical operations.

The number of devices connected to the Internet is currently just under nine billion. In the next two years, it is expected to grow to 20.5 billion worldwide [17]. The devices at the edge, i.e. at the edge of the network, will grow most of all. This is also expected by the process industry-albeit with much smaller overall figures. There is much to be said for processing the rapidly growing volumes of data on the spot and only exchanging abstract and reduced data centrally. This is called Edge Computing. Analytical systems with high demands on real-time capability, such as process spectrometers in millisecond intervals, can only evaluate spectra with on-board embedded systems.

Virtualized operating systems have long been common in the business sector, especially for servers. A virtualization layer is placed between the physical hardware (PC, PLC, etc.) and its logical parts (operating system, application) so that the logical parts are no longer dependent on the hardware. One speaks of virtual machines (VM). This means that software can no longer run locally on one computer, but on computer networks- even distributed over the Internet as virtual appliances.

The use of templates or the fast cloning of virtualized applications is a very important advantage of software virtualizations. It opens up new backup options in accordance with compliance policies and greatly simplifies the recovery of guest operating systems. A validated state can be restored in the system in a few minutes by restoring the last validated version, for example if functional tests after a software patch or firmware renewal cause problems. This would never be possible for individual applications without completely re-equipping a system to the last validated state, and in the GMP environment there is the dilemma of patch or compliance.

The use of software virtualization has long been used at higher levels of automation (cloud computing for ERP, MES or SCADA systems). It is also highly interesting for automation in the fog or edge area, although there is still less experience here. The increasing implementation of process control functionalities and advanced process control on virtual machines would be a great advantage and could reach even further into the edge in the future.

The definition of open and at the same time secure interfaces is one of the biggest and most urgent tasks of digitalization, without which it cannot continue. It is not enough to refer abstractly to the growth of data protection guidelines and to expect IT security to continue to evolve automatically with the challenges.

In order to define a secure data transmission, basic requirements and business models must now be established, because without these no secure architecture can be designed. Some conflicting requirements are clearly set out: The legal requirement for almost always the highest IT security standards to protect the infrastructure of the process industry; the desire for a runtime of twenty years, for high flexibility as a benefit of new technologies and for reasonable installation and maintenance costs. There is an unpredictable arms race of currently considered safe procedures against the weapons of hackers around the world. Only an open mechanism that provides for permanent hardware and software extensions would be able to fulfil the requirements.

\section{Conclusions}

Implementing new digital technologies requires building new skills, investing in talent, and significant change management to pick up the entire organization. The process industry is facing changes that will take place over a long period of time and require a pronounced need for research and design. Digital transformation is not possible on its own, it can only be carried out together with all those involved.

Funding: This research received no external funding. 
Acknowledgments: The author would like to thank many experts-in particular the NAMUR AKs 3.6, 3.6.3, and 3.7 as well as his BAM colleagues-for the interesting discussions which contributed to the picture presented. The author thanks Klas Meyer for valuable support in technical discussions and proofreading.

Conflicts of Interest: The author declares no conflict of interest.

\section{References}

1. Gransche, B.; Shala, E.; Kimpeler, S.; Hubig, C.; Alpsancar, S.; Harrach, S. Change of autonomy and control through new human-technology interactions. In Fundamental Issues of Autonomous Human-Technology Relationships; Fraunhofer Institute ISI: Stuttgart, Germany, 2014.

2. ProcessNet Fachgemeinschaft Prozess-, Apparate- und Anlagentechnik (PAAT)—Survival Strategies for the Process Industry. Workshop data concepts, data analysis, big data and artificial intelligence. In Tutzing Symposium 2018. 100\% Digital! DECHEMA e.V.: Tutzing, Germany, 2018.

3. Lee, E.A.; Seshia, S.A. Introduction to Embedded Systems-A Cyber-Physical Systems Approach, 2nd ed.; MIT Press: Cambridge, MA, USA, 2017.

4. ZVEI-Zentralverband Elektrotechnik- und Elektronikindustrie e. V. Module-Based Production in the Process Industry -Effects on Automation in the "Industrie 4.0" Environment; White Paper; ZVEI: Frankfurt, Germany, 2015; Available online: https://www.zvei.org/en/press-media/publications/white-paper-module-basedproduction-in-the-process-industry/ (accessed on 23 December 2019).

5. Hiwale, R.S.; Bhate, N.V.; Mahajan, Y.S.; Mahajani, S.M. Industrial applications of reactive distillation: recent trends. Int. J. Chem. React. Eng. 2004, 2. [CrossRef]

6. Taylor, R.; Krishna, R. Modelling reactive distillation. Chem. Eng. Sci. 2000, 55, 5183-5229. [CrossRef]

7. Lipnizki, F.; Field, R.W.; Ten, P.-K. Pervaporation-based hybrid process: A review of process design, applications and economics. J. Memb. Sci. 1999, 153, 183-210. [CrossRef]

8. Lutze, P.; Gorak, A. Reactive and membrane-assisted distillation: Recent developments and perspective. Chem. Eng. Res. Des. 2013, 91, 1978-1997. [CrossRef]

9. Segovia-Hernández, J.G.; Hernández, S.; Bonilla Petriciolet, A. Reactive distillation: A review of optimal design using deterministic and stochastic techniques. Chem. Eng. Process. Process Intensif. 2015, 97, 134-143. [CrossRef]

10. Kiss, A.A. Taking Reactive Distillation to the Next Level of Process Intensification. Chem. Eng. Trans. 2018, 69, 553-558.

11. Muthia, R.; Reijneveld, A.G.T.; Van Der Ham, A.G.J.; Ten Kate, A.J.B.; Bargeman, G.; Kersten, S.R.A.; Kiss, A.A. Novel method for mapping the applicability of reactive distillation. Chem. Eng. Process. Process Intensif. 2018, 128, 263-275. [CrossRef]

12. VDI/VDE-Gesellschaft Mess- und Automatisierungstechnik (GMA) and NAMUR-Interessen-ge-meinschaft Automatisierungstechnik der Prozessindustrie. Technology Roadmap Process Sensors 4.0—Requirements for Future Automation Concepts; VDI/VDE-GMA: Düsseldorf, Germany, 2015.

13. OPC UA. Available online: http://www.opcfoundation.org (accessed on 25 October 2019).

14. Ladwig, B. The smart laboratory needs a communication standard. In Trend Report 2018 Analysis, Biotechnology and Laboratory Technology; SPECTARIS, German Industry Association for Optical, Medical and Mechatronic Technologies e.V.: Berlin, Germany, 2018.

15. The Reference Architecture Model RAMI 4.0 and the Industry 4.0 Component. Available online: https://www.zvei.org/en/subjects/industrie-4-0/the-reference-architectural-model-rami-40-and-theindustrie-40-component (accessed on 23 December 2019).

16. Baran, P. On Distributed Communications; Document RM-3420-PR; Rand Corporation: Santa Monica, CA, USA, 1964.

17. Schmieder, J. Recken am Rand; Süddeutsche Zeitung: München, Germany, 2018. 